
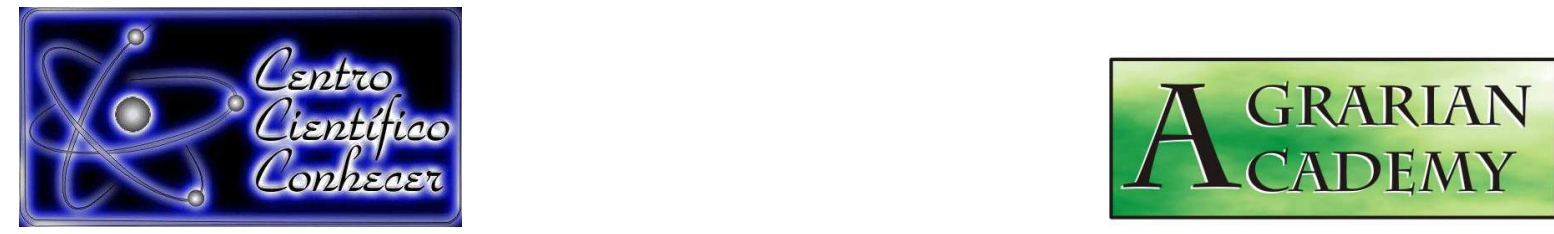

\title{
ESTUDO DA VIABILIDADE POLÍNICA DE Myrciaria cauliflora (Mart.) O. Berg. POR MEIO DE DISTINTOS MÉTODOS DE COLORAÇÃO
}

Leila Pereira Neves Ramos ${ }^{1}$, Vera Lúcia Simões André ${ }^{1}$, Vanessa dos Santos de Mello $^{2}$, Jenifer Fernanda Damasio ${ }^{3}$, Isane Vera Karsburg ${ }^{4}$

${ }^{1}$ Graduada em Licenciatura Plena em Ciências Biológicas, Universidade do Estado de Mato Grosso, Campus de Alta Floresta, MT - Brasil

${ }^{2}$ Bióloga, Mestranda pelo Programa de Pós Graduação em Genética e Melhoramento de Plantas, Universidade do Estado de Mato Grosso, Campus de Alta Floresta, MT - Brasil ${ }^{3}$ Mestre em Genética e Melhoramento de Plantas, Universidade do Estado de Mato Grosso, Alta Floresta, MT - Brasil

${ }^{4}$ Docente da Faculdade de Ciências Biológicas e Agrárias, Universidade do Estado de Mato Grosso. 78.580-000 Alta Floresta, MT - Brasil leila_pereiraramos@hotmail.com

Recebido em: 30/11/2017 - Aprovado em: 15/12/2017 - Publicado em: 31/12/2017 DOI: 10.18677/Agrarian_Academy_2017b15

\begin{abstract}
RESUMO
A jabuticaba (Myrciaria cauliflora) é originária do Brasil, nativa da Mata Atlântica e pertencente à família Myrtaceae. É uma fruta considerada rica em vitaminas, antocianinas, fibras e sais minerais. $O$ presente estudo objetivou-se em estimar a viabilidade polínica de três populações de $M$. cauliflora existente no município de Alta Floresta - MT. Para o estudo foram utilizados a solução de 2,3,5 trifeniltetrazolio (TTC) em duas concentrações $(0,075 \%$ e $0,30 \%)$ sendo o material exposto em quatro tempos diferentes e o corante Lugol $2 \%$. Na preparação das lâminas com 0 uso do TTC, adicionou-se uma gota de cada tratamento sobre a lâmina, em seguida sendo coberto com uma lamínula. Já para o teste com Lugol $2 \%$, foi utilizada técnica de esmagamento das anteras na elaboração das lâminas. $\mathrm{Na}$ estimativa da viabilidade polinica das populações 01,02 e 03 de $M$. cauliflora, os testes colorimétricos (2,3,5 trifeniltetrazolio (TTC) e Lugol $2 \%$ ), se mostraram capazes de distinguir os pólens viáveis dos inviáveis, mostrando-se eficazes ao estimar a viabilidade polínica desta espécie. Diante das médias observou-se um aumento da viabilidade polínica no decorrer dos tempos de exposição do material na solução do TTC. A temperatura de $30^{\circ} \mathrm{C}$ apresentou as maiores médias de viabilidade polínica, em comparação com a de $21^{\circ} \mathrm{C}$. Os índices de viabilidade polínica de $M$. cauliflora estimada pelo corante Lugol $2 \%$ mostraram-se satisfatórias (médias/altas).
\end{abstract}

PALAVRAS-CHAVE: corante, jabuticaba, pólens. 


\title{
STUDY OF THE POLYNICAL VIABILITY OF Myrciaria cauliflora (Mart.) O. Berg. BY DIFFERENT COLORING METHODS
}

\begin{abstract}
The jabuticaba (Myrcia cauliflora) is native to Brazil, native to the Atlantic Forest and belongs to the Myrtaceae family. It is a fruit considered rich in vitamins, anthocyanins, fibers and minerals. The present study aimed to estimate the pollen viability of three populations of M. cauliflora in the municipality of Alta Floresta - MT. For the study, 2,3,5-triphenyltetrazolium (TTC) solution was used in two concentrations $(0.075 \%$ and $0.30 \%$ ), the material being exposed at four different times and the Lugol dye $2 \%$. In the preparation of the slides using triphenyltetrazolium (TTC), a drop of each treatment was added on the slide, then covered with a cover slip. For the test with Lugol $2 \%$, a basic technique of crushing the anthers in the preparation of the slides was used. In the estimation of pollen viability of populations 01,02 and 03 of $\mathrm{M}$. cauliflora, the colorimetric tests (2,3,5 triphenyltetrazolium (TTC) and Lugol $2 \%$ ) were able to distinguish viable pollen from non-viable pollens, effective in estimating the pollen viability of this species. In view of the averages, an increase in the pollen viability during the exposure times of the material in the TTC solution was observed. The temperature of $30^{\circ} \mathrm{C}$ presented the highest averages of pollen viability compared to $21^{\circ} \mathrm{C}$. The pollen viability indexes of $\mathrm{M}$. cauliflora estimated by Lugol dye $2 \%$ were satisfactory (medium / high).
\end{abstract}

KEYWORDS: dye, jabuticaba, pollen

\section{INTRODUÇÃO}

A jabuticaba (Myrciaria cauliflora) originária do Brasil é pertencente à família Myrtaceae. São conhecidas em média nove espécies de jabuticabeira (DANNER, et al., 2011). As jabuticabas se diferenciam sob vários aspectos morfológicos das folhas, flores e frutos (LORENZI et al., 2006).

A $M$. cauliflora é uma fruta considerada rica em vitaminas, antocianinas, fibras e sais minerais. Esses nutrientes estão em maior concentração na casca do fruto. Uma opção para o consumo desses nutrientes é a incorporação das cascas da jabuticaba em alimentos, principalmente por ser considerado um corante natural que apresenta elevados teores de antocianinas que são benéficas para a saúde (FERREIRA et al., 2012). Existe um grande interesse por parte das indústrias farmacêuticas e alimentícias, pois a jabuticabeira contém um alto teor de óleos essenciais presentes em suas folhas (APEL et al., 2006).

Mesmo que os polinizadores contribuam para a reprodução das espécies das plantas, diversos fatores podem afetar a reprodução das espécies. Pois a viabilidade, pode ser afetada, por exemplo por: dessecação, baixa umidade relativa, disponibilidade de substância de reserva, idade da flor, metabolismo, alterações morfológicas da antera, temperatura entre outros fatores ocorrentes (DAFNI et al., 2000).

O estudo por meio da viabilidade polínica é responsável pela seleção de genótipos para o melhoramento das espécies (CABRAL et al., 2013). Portanto a análise dos grãos de pólen através do uso de corantes permite a determinação de sua viabilidade polínica (HISTER et al., 2016). Dentre as técnicas de citogenética, o estudo de viabilidade polínica quando empregada em programas de melhoramento, fornece subsídios na tomada de decisão para o melhoramento das espécies (BRAMBATTI et al., 2016). 
Podem ser utilizados testes, dentre os quais uma opção é a utilização do teste com cloreto de 2,3,5 trifeniltetrazólio (TTC) (RAMOS et al., 2006). O TTC é importante para otimização da aplicação dos recursos técnicos nos laboratórios, possibilitando a análise de amostras de espécies de custos razoáveis mostrando-se eficiente (SILVA et al., 2013). Um aspecto importante é o uso da solução do trifeniltetrazólio (TTC) na concentração adequada (BRASIL, 1992). O Lugol também é utilizado em estudos como testes da viabilidade polínica de diversas espécies (SOUZA et al., 2002). Mostra-se eficiente e aponta com precisão resultados de viabilidade polínica (LIMA et al., 2015).

O presente trabalho teve como objetivo estimar a viabilidade polínica de Myrciaria cauliflora por meio de testes colorimétricos, usando os corantes de 2,3,5 trifeniltetrazolio (TTC) em quatro tempos diferentes e o Lugol $2 \%$, avaliando a eficiência dos corantes sobre a espécie. Este estudo poderá contribuir fornecendo informações e dados sobre a viabilidade polínica que vem a auxiliar nos processos de melhoramento da espécie estudada.

\section{MATERIAL E MÉTODOS}

O experimento foi realizado no Laboratório de Citogenética e Cultura de Tecidos Vegetais na Universidade do Estado de Mato Grosso (UNEMAT) do Campus de Alta Floresta-MT, localizado no Bairro Jardim Flamboyant. Foram coletados 30 botões florais de $M$. cauliflora em estágio de pré-antese no município de Alta Floresta - MT (Tabela 1).

TABELA 1. Identificação dos acessos, local de coleta dos botões florais em préantese das populações de Myrciaria cauliflora (Mart.) O. Berg. e os dados de GPS.

\begin{tabular}{ccc}
\hline População & Local de Coleta & Coordenadas \\
\hline Pop.1 & Alta Floresta - MT & Sul: 0952'17,50 "Oeste: 560ㄴ'82,80" \\
Pop. 2 & Alta Floresta - MT & Sul: 0951'14,30 "Oeste: 560'63,30" \\
Pop. 3 & Alta Floresta - MT & Sul: 0951'45,40 "Oeste: 5604'15,22" \\
\hline
\end{tabular}

Para o teste de 2,3,5 (TTC) trifeniltetrazolio, os botões florais foram cortados transversalmente com o auxílio de um bisturi, para a retirada das anteras, maceradoas com um bastão de vidro para a liberação dos grãos de pólen na solução de TTC com duas concentrações $(0,075 \%$ e $0,30 \%)$ permanecendo em quatro horários diferentes de exposição ao corante (6:00, 12:00, 18:00 e 24:00 horas). O material foi conduzido em ambiente com temperatura controlada $\left(21^{\circ} \mathrm{C}\right.$ e $\left.30^{\circ} \mathrm{C}\right)$.

Na preparação das lâminas adicionou-se uma gota de cada tratamento sobre cada lâmina, após o material foi coberto com uma lamínula. Para a análise das lâminas adotou-se o método de varredura, contabilizando 300 grãos de pólen por lâmina, fazendo cinco repetições cada, totalizando 1.500 grãos de pólens para cada tratamento observados sob microscópio óptico na objetiva de 40x. A viabilidade polínica foi determinada pela capacidade de coloração dos grãos de pólen, considerando viáveis os pólens que apresentaram coloração da exine e da intine ou protoplasma de vermelho e inviáveis aqueles que não apresentaram coloração.

Para estimar a viabilidade polínica por meio do teste colorimétrico, foram utilizados botões florais em pré-antese, das três populações de $M$. cauliflora, foi testado a eficiência do corante, Lugol $2 \%$, na estimativa da viabilidade polínica da espécie. Para esse corante, a viabilidade foi determinada pela capacidade de auto coloração dos grãos de pólen, onde foram considerados viáveis os pólens que 
apresentaram coloração da exina e da intina ou protoplasma bem definido e inviáveis aqueles que apresentaram exina corada e ausência de protoplasma (cor).

No processo de preparo das lâminas as anteras foram cortadas transversalmente com o auxílio do bisturi. Para a liberação dos grãos de pólen, as anteras foram maceradas com bastão de vidro, sobre uma gota de lugol $2 \%$. Em seguida, o material foi coberto com uma lamínula e observado ao microscópio. Com o objetivo obter amostragens ao acaso dos grãos de pólen corados, foi utilizou-se o método de varredura, contabilizando 300 grãos de pólens por lâmina com 5 repetições cada, com o total de 1500 grãos de pólen para o corante testado. As lâminas foram observadas no microscópio óptico na objetiva de 40x.

Os dados foram submetidos à comparação de médias pelo teste de tukey em nível de $5 \%$ de probabilidade pelo programa $R$, versão 3.3.2 (R CORE TEAM, 2016), com o auxílio do pacote ExpDes, versão 1.1.2 (FERREIRA et al., 2013). Os dados do corante lugol foram representados de forma gráfica realizados no software SigmaPlot (versão 11).

\section{RESULTADOS E DISCUSSÃO}

Diante do estudo de viabilidade polínica com as três populações de Myrciaria cauliflora (Tabela 2), pode-se observar que as concentrações de 2,3,5 de cloreto de Trifeniltetrazólio (TTC), apresentaram diferença significativa para as duas temperaturas testadas. A temperatura de $30 \stackrel{\circ}{ } \mathrm{C}$ apresentou as maiores médias de viabilidade polínica, quando comparado com a de $21^{\circ} \mathrm{C}$.

De acordo com Soares et al. (2011) ao desenvolverem um trabalho sobre viabilidade polínica, obtiveram resultados semelhantes a estes estudo, onde a temperatura de $25{ }^{\circ} \mathrm{C}$ foi mais adequada para a espécie de Ananas macrodontes, e $30^{\circ} \mathrm{C}$ para Ananas comosus var. bracteatu. VARA PRASAD et al. (1999) relata que, fatores tais como umidade relativa, temperatura, pressão osmótica do conteúdo celular do pólen e resistência da parede polínica pode influenciar na viabilidade polínica da espécie.

TABELA 2. Viabilidade polínica de Myrciaria cauliflora (Mart.) O. Berg., coloração com o uso de TTC na concentração de $0,075 \%$ exposto em duas temperaturas.

\begin{tabular}{|c|c|c|}
\hline Populações & Temperaturas & Viabilidade polínica (\%) \\
\hline \multirow{2}{*}{01} & $21^{\circ} \mathrm{C}$ & $37,33 b$ \\
\hline & $30{ }^{\circ} \mathrm{C}$ & $42,99 a$ \\
\hline \multirow{2}{*}{02} & $21^{\circ} \mathrm{C}$ & $40,75 b$ \\
\hline & $30 \stackrel{\circ}{C}$ & $47,22 a$ \\
\hline \multirow{2}{*}{03} & $21^{\circ} \mathrm{C}$ & $42,74 b$ \\
\hline & $30{ }^{\circ} \mathrm{C}$ & $49,16 a$ \\
\hline CV (\%) & & ,41 \\
\hline
\end{tabular}

Médias seguidas pela mesma letra maiúscula nas linhas e minúscula nas colunas não diferem pelo teste de tukey a nível de $5 \%$ de significância.

Observa-se na tabela 3 que as médias diferenciaram-se significativamente entre si. Assim como para a concentração de $0,075 \%$ a temperatura de $30^{\circ} \mathrm{C}$ apresentou as maiores médias de viabilidade polínica, exceto para a população 03, a qual não se diferenciou da temperatura de $30^{\circ} \mathrm{C}$. 
Leite et al. (2015) em um estudo com a espécie Tabebuia roseoalba utilizando a concentração de $0,075 \%$, obtiveram resultados com um crescente número de pólens viáveis com o aumento do tempo de exposição sendo o mais indicado para estimar a viabilidade da espécie o tratamento de 72 horas, além de apresentar melhor distinção na coloração dos pólens.

Santos et al. (2014) nas análises de viabilidade polínica com a espécie Passiflora setacea, relataram que se for adotado a menor concentração $(0,075 \%)$, em estudos futuros de viabilidade polínica, proporcionará a redução de custos nos estudos de melhoramento de espécies.

TABELA 3. Viabilidade polínica de Myrciaria cauliflora (Mart.) O. Berg., coloração com o uso de TTC na concentração de $0,30 \%$ exposto em duas temperaturas.

\begin{tabular}{|c|c|c|}
\hline Populações & Temperaturas & Viabilidade polínica (\%) \\
\hline \multirow{2}{*}{01} & $21^{\circ} \mathrm{C}$ & $55,58 \mathrm{~b}$ \\
\hline & $30^{\circ} \mathrm{C}$ & $62,49 a$ \\
\hline \multirow{2}{*}{02} & $21^{\circ} \mathrm{C}$ & $58,50 \mathrm{~b}$ \\
\hline & $30 \stackrel{\circ}{\circ}$ & $64,99 a$ \\
\hline \multirow{2}{*}{03} & $21^{\circ} \mathrm{C}$ & $60,41 a$ \\
\hline & $30 \stackrel{\circ}{\circ}$ & $63,00 \mathrm{a}$ \\
\hline CV (\%) & & 0,84 \\
\hline
\end{tabular}

Médias seguidas pela mesma letra maiúscula nas linhas se minúscula nas colunas Não diferem pelo teste de tukey a nível de $5 \%$ de significância

Com relação aos tempos de exposição dos grãos de pólen, nas concentrações, verificou-se que os tratamentos de forma geral não diferiram entre si. Pelas médias observa-se o aumento da viabilidade polínica no decorrer dos tempos de exposição, porém em 24 horas obteve-se o maior percentual de viabilidade dos pólens, mostrando-se como o horário mais adequado para a exposição dos pólens para que adquiram coloração máxima (Tabela 4).

Lauton et al. (2016) fizeram um trabalho de estimativa da viabilidade polínica de Jasmim manga testando a solução de tetrazólio (TTC) nos respectivos períodos de $6,12,18$ e $24 \mathrm{~h}$, onde o período de $12 \mathrm{~h}$ com a concentração de $0,075 \%$ demonstrou-se como o melhor percentual de grãos viáveis da espécie.

TABELA 4. Percentuais da viabilidade dos grãos de pólen de Myrciaria cauliflora (Mart.) O. Berg., durante os tempos de exposição no corante trifeniltetrazolio (TTC).

\begin{tabular}{cc}
\hline Tempos de exposição (horas) & Viabilidade polínica (\%) \\
\hline 6 & $46,94 \mathrm{c}$ \\
12 & $49,80 \mathrm{bc}$ \\
18 & $54,08 \mathrm{ab}$ \\
24 & $57,13 \mathrm{a}$ \\
\hline CV (\%) & 20,95 \\
\hline
\end{tabular}

Médias seguidas pela mesma letra maiúscula nas linhas e minúscula nas colunas não diferem pelo teste de tukey a nível de 5\% de significância. 
As médias de viabilidade polínica estimada pelo corante lugol $2 \%$ mostraram-se altas para as populações $02 \mathrm{com} 72,66 \%$ e a $03 \mathrm{com} 77,00 \%$, já a população 01 apresentou um percentual médio com $67,66 \%$ de pólens corados (Figura 1).

Neto et al., (2006) utilizando lugol 1\% para estimar a viabilidade polínica de populações de Solanum paniculatum, Santos et al., (2016) em estudos em Costus spiralis, Nascimento et al., (2014) utilizaram o lugol para estimar a viabilidade polínica de Delonix regia, Souza et al., (2014) estimaram a viabilidade polinica de espécies do gênero, Tabebuia e Ramos et al. (2017) também obtiveram resultados eficientes utilizando o Lugol $2 \%$ ao testar a viabilidade polínica da espécie Momordica charantia, com médias acima de $80 \%$.

Cabral et al. (2013) constataram em um estudo com a espécie Theobroma cacao, que o corante lugol apresentou melhor distinção dos pólens viáveis dos inviáveis, proporcionando dados mais apurado na diferenciação de coloração dos pólens mostrando-se eficiente. Segundo Souza et al. (2002) a viabilidade polínica é considerada alta para valores que são acima de $70 \%$.

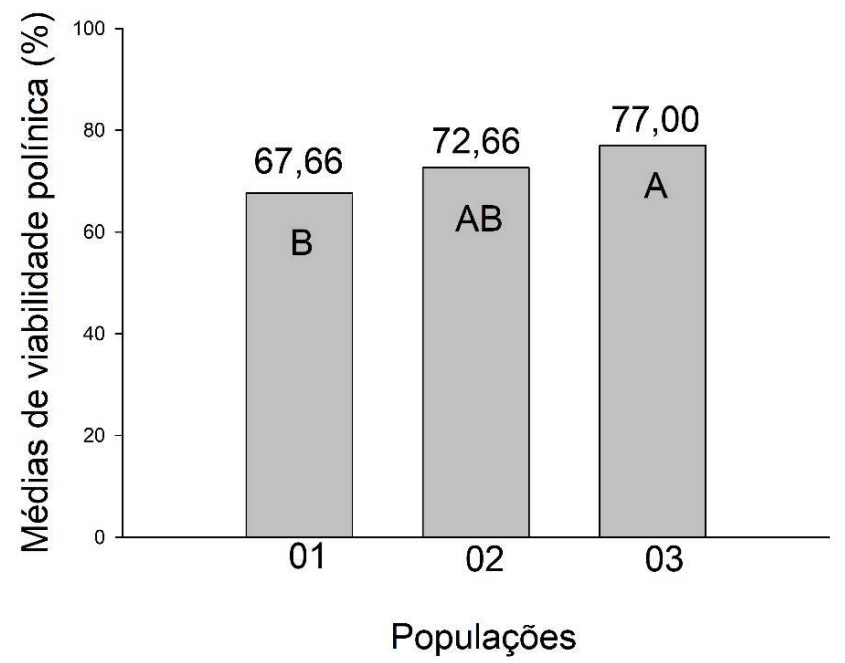

FIGURA 1. Médias percentuais de viabilidade polínica entre as populações de Myrciaria cauliflora (Mart.) $\mathrm{O}$. Berg., estimada pelo corante Lugol 2\%. CV (\%) $=9,16$.

No uso dos corantes TTC $(0,30$ e $0,075 \%)$ e o uso do lugol $2 \%$ se mostraram eficientes quanto a diferenciação da coloração dos grãos de pólen viáveis, corados de forma intensa e os inviáveis sem coloração ou com protoplasma reduzido no grão de pólen. Estes dados corroboram com informações apresentadas por Gibbert et al., (2017) no estudo da viabilidade polínica de três populações de Syzygium malaccense em que os dados de coloração com TTC e de lugol $2 \%$ se relacionam quanto ao número de grãos de pólen viáveis e inviáveis. Contudo, qualquer um dos corantes podem ser utilizados para estas espécies pertencentes a família Myrtaceae. 


\section{CONCLUSÃO}

De acordo com os dados obtidos, pode-se concluir que nas populações de M. cauliflora os testes colorimétricos 2,3,5 trifeniltetrazolio (TTC) e o Lugol $2 \%$, mostraram-se eficazes para estimar a viabilidade polínica desta espécie.

Diante das médias observou-se um aumento da viabilidade polínica no decorrer dos tempos de exposição do material na solução do TTC. A temperatura de $30 \stackrel{\circ}{ } \mathrm{C}$ apresentou as maiores médias de viabilidade polínica, em comparação com a de $21^{\circ} \mathrm{C}$. Os índices de viabilidade polínica de M. cauliflora estimada pelo corante Lugol 2\% mostraram-se médias e altas.

\section{AGRADECIMENTOS}

Os autores agradecem à Universidade do Estado de Mato Grosso, (UNEMAT) Alta Floresta - MT.

\section{REFERÊNCIAS}

APEL, M. A.; SOBRAL, M.; ZUANAZZI, J. A.; HENRIQUES, A. T. Essential oil composition of four Plinia species (Myrtaceae). Flavour and Fragrance Journal, Chichester, v. 21, p. 565-567, 2006. Disponível em: $<$ http://onlinelibrary.wiley.com/doi/10.1002/ffj.1638/full>. doi: $10.1002 / f f j .1638$

BRAMBATTI, A.; BRAMMER, S. P.; WIETHÖLTER, P.; JUNIOR, A. do N. Estabilidade genética em triticale estimada pela viabilidade polínica. Arquivos do Instituto Biológico, v. 83, p. 01-07, 2016. Disponível em: <http://www.scielo.br/pdf/aib/v83/1808-1657-aib-83-e0802014.pdf>.

doi: 10.1590/1808-1657000802014

BRASIL. Ministério da Agricultura e Reforma Agrária. Regras para análise de sementes. Brasília: SNDA/DNPV/CLAV, 1992. 365p. Disponível em: $<$ http://www.scielo.br/scielo.php?script=sci_nlinks\&ref=000080\&pid=S0101$3122200500020002400007 \&$ Ing=en>.

CABRAL, J. C.; ROSSI, A. A. B.; KLEIN, M. E.; VIEIRA, F. S.; GIUSTINA, L. D. Estimativa da viabilidade polínica em acessos de Theobroma cacao L. baseada em testes colorimétricos. Enciclopédia Biosfera, v.9, n.17, p.2780-2788, 2013. Disponível

em: <http://www.conhecer.org.br/enciclop/2013b/CIENCIAS\%20BIOLOGICAS/estimativa $\% 20$ da\%20viabilidade.pdf $>$.

DAFNI, A. FIRMAGE, D. Pollen viability and longevity: practical, ecological and evolutionary implications. Plant Systematics and Evolution. 222: 113-132, 2000. Disponível em: <https://link.springer.com/article/10.1007/BF00984098>.

DANNER, M. A.; CITADIN, I.; SASSO, S. A. Z.; SACHET, M. R.; MALAGI, G. Modo de reprodução e viabilidade de pólen de três espécies de jabuticabeira. Revista Brasileira de Fruticultura, v. 33, n. 2, p. 345-352, 2011. Disponível em: $<$ http://www.scielo.br/pdf/rbf/v33n2/aop06211>. 
FERREIRA, E.B.; CAVALCANTI, P.P.; NOGUEIRA, D. A. ExpDes: Experimental Designs package. $R$ package version 1.1.2. 2013.

FERREIRA, A. E., FERREIRA, B. S., LAGES, B., Maria, M., RODRIGUES, F., Ayala, V.; VILLELA, N. A. produção, caracterização e utilização da farinha de casca de jabuticaba em biscoitos tipo cookie. Alimentos e Nutrição, v. 23, n. 4, p. 603-607, 2012. Disponível em:

<http://serv-bib.fcfar.unesp.br/seer/index.php/alimentos/article/viewFile/603/2017>.

GIBBERT, L. F.; RIBEIRO, R.; FERNANDES, S. C.; MELLO, V. dos S. de.; KARSBURG, I. V. Viabilidade polinica de Syzygium malaccense por métodos colorimétricos. Agrarian Academy, Centro Científico Conhecer - Goiânia, v.4, n.7; p. 2017. Disponível em: <http://www.conhecer.org.br/Agrarian\%20Academy/2017a/viabilidade.pdf>. doi: 10.18677/Agrarian_Academy_2017a45

HISTER, C. A. L.; TEDESCO, S. B. Estimativa da viabilidade polínica de araçazeiro (Psidium cattleianum Sabine) através de distintos métodos de coloração. Revista brasileira de plantas medicinais, v. 18, n. 1, p. 135-141, 2016. Disponível em: <https://www.researchgate.net/profile/Carmine Hister2/publication/301572636 Estim ativa_da_viabilidade_polinica_de_aracazeiro_Psidium_cattleianum_Sabine_atraves de_distintos_metodos_de_coloracao/links/576-da40608ae842225a40a4d/Estimativada-viabilidade-polinica-de-aracazeiro-Psidium-cattleianum-Sabine-atraves-dedistintos-metodos-de-coloracao.pdf>. doi.org/10.1590/1983-084X/15_081.

LAUTON, D. S.; SANTOS, A. C.; KARSBURG, I. V.; DAMASIO, J. F.; Viabilidade polínica de Jasmim Manga com corante tetrazólio. Ciência \& Tecnologia: Fatec-JB, Jabuticabal, v. 8, n.1, 2016. Disponível em:

$<$ www.citec.fatecjab.edu.br/index.php/files/article/download/1057/pdf>.

LEITE, D. M.; CARDOSO.; M. A.; KARSBURG, I. V. Viabilidade polínica de Tabebuia roseoalba (Ridl.) Sandwith com 2,3,5 trifenil tetrazolio. I Congresso Florestal de Mato Grosso, Sinop-MT, $2015 . \quad$ Disponível em: <file:///C:/Users/Administrador/Downloads/OCORRENCIA-DE-CHRYSOMELIDAEEUMOLPINAE-E-DESCRICAO-DE-DANOS-EM-Tachigali-vulgaris-FABACEAE-EMSINOP-MT-pg115.pdf>.

LIMA, J. dos S.; ROCHA, V. D. da.; TIAGO, A. V.; SANTOS, T. A. dos.; ROSSI, A. A. $B$. Influência do horário de coleta sobre a viabilidade de grãos de pólen em acerola (Malpighia emarginata DC.). Enciclopédia Biosfera, Centro Científico Conhecer Goiânia, v.11 n.21; p. 3216, 2015. Disponível em: <http://www.conhecer.org.br/enciclop/2015b/multidisciplinar/influencia\%20do\%20hor ario.pdf $>$.

LORENZI, H.; BACHER, L.; LACERDA, M.; SARTORI, S. Frutas brasileiras e exóticas cultivadas: de consumo in natura. São Paulo: Instituto Plantarum de Estudos da Flora, 2006. 672p. 
NASCIMENTO, L. S. do.; BENEVENUTI, A. S.; LEITE, D. M.; SILVA, D. D. da.; Moura, E. A.; MIRANDA, D.; KARSBURG, I. V. Estimativa da Viabilidade Polínica e Índice Meiótico de" Delonix Regia". Estudos, v. 41, p. 83-88, 2015. No texto consta 2014.

NETO, OTÁVIO DIAS SANTOS; KARSBURG, I. V.; YOSHITOME, M. Y. Viabilidade e germinabilidade polínica de populações de jurubeba (Solanum paniculatum I.). Revista de Ciências Agro-Ambientais, Alta Floresta, v. 4, n. 1, p. 67-74, 2006. Disponível em: <http://www.unemat.br/revistas/rcaa/docs/vol4/8_artigo_v4.pdf>.

$R$ Core Team. R: A language and environment for statistical computing. $R$ Foundation for Statistical Computing, Vienna, Austria. URL https://www.R project.org/. 2016.

RAMOS, L. P. N.; ANDRÉ, V. L. S.; MELLO, V. dos S. de.; WINK, F.; KARSBURG, I. V. Estimativa da viabilidade polínica do Melão-de-São-Caetano (Momordica charantia L.) com diferentes métodos de coloração. Agrarian Academy, Centro Científico Conhecer - Goiânia, v.4, n.7; p. 2017. Disponível em: <http://www.conhecer.org.br/Agrarian\%20Academy/2017a/estimativa\%20da\%20viabi lidade.pdf>.doi: 10.18677/Agrarian_Academy_2017a23

RAMOS, T. Z.; PIZZOLITTO, E. L.; PIZZOLITTO, A. C. Uso do teste com cloridrato de trifenil tetrazólio (CTT) para detecção de bacteriúria sintomática e assintomática. RBAC, v. 38, n. 3, p. 197-199, 2006. Disponível em: <https://www.researchgate.net/profile/Elisabeth_Pizzolitto/publication/237500559_Us o_do_teste_com_cloridrato_de_trifenil_tetrazolio_CTT_para_deteccao_de_bacteriuri a_sintomatica_e_assintomatica_Use_of_the_triphenil_tetrazolium_chloride_TTC_tes t_to_detect_symptomatic_and_asymptomati/links/53ff- $24360 \mathrm{cf} 23 \mathrm{~b} \overline{\mathrm{b}} 019 \mathrm{bef} 6 \overline{\mathrm{b}} \mathrm{b} / \mathrm{Uso}$ do-teste-com-cloridrato-de-trifenil-tetrazolio-CTT-para-deteccao-de-bacteriuriasintomatica-e-assintomatica-Use-of-the-triphenil-tetrazolium-chloride-TTC-test-todetect-symptomatic-and-asymptomati.pdf>.

SANTOS, B. N. V. dos.; MACEDO, W. de A.; MELLO, V dos S. de.; DAMASIO, J. F.; SANTOS, L. C. B. dos.; LEITE, D. M.; KARSBURG, I. V. Estimativa da viabilidade dos grãos de pólen de cana-do-brejo baseada em distintos métodos de coloração. Ciência \& Tecnologia: Fatec-JB, Jaboticabal, v. 8, n.1, 2016. Disponível em: <http://www.citec.fatecjab.edu.br/index.php/files/article/view/990>.

SANTOS, M. L.; PORTO, A. C. M.; OlIVEIRA, A. C. Avaliação da viabilidade de embriões de plantas matrizeiras de maracujazeiro 'do sono' (Passiflora setacea DC) contrastantes quanto à capacidade de germinação. XX ENGENE - Encontro de Genética do Nordeste. Campina Grande - Paraíba - Brasil, 2014. Disponível em: $<$ file:///C:/Users/Administrador/Downloads/2548-7331-1-PB.pdf>

SILVA, R. C. de.; SOUZA, G, C. R. de.; de BARROS, F. N., J.; PANOBIANCO, M. Adaptação do teste de tetrazólio para avaliação da viabilidade e do vigor de sementes de girassol. Pesquisa Agropecuária Brasileira, v. 48, n. 1, p. 105-113, 2013. Disponível em: <http://www.scielo.br/pdf/pab/v48n1/14.pdf>.

doi: 10.1590/S0100-204X2013000100014 
SOARES, T. L.; SOUZA, E. H. de, E.; ROSSI, M. L.; SOUZA, F. V. D. Morfologia e viabilidade de grãos de pólen de acessos silvestres de abacaxi. Ciência Rural, v. 41, n. 10, 2011. Disponível em: <http://www.redalyc.org/html/331/33119857006/>.

SOUZA, M. D. A. de.; SILVEIRA, G. F. da.; SILVA, M. de S. A. da.; KARSBURG, I. V. Estimativa da viabilidade polínica em indivíduos de Tabebuia impetiginosa e Tabebuia chrysotricha (Mart. ex. DC.) Standl. (Bignoniaceae) através de métodos citoquímicos. Enciclopédia Biosfera, Centro Científico Conhecer - Goiânia, v.10, n.18; $\quad$ p. $2014 . \quad$ Disponível em: <http://www.conhecer.org.br/enciclop/2014a/MULTIDISCIPLINAR/estimativa.pdf>.

SOUZA, M. M.; PEREIRA, T. N. S.; MARTINS, E. R. Microsporogênese e microgametogênese associadas ao tamanho do botão floral e da antera e viabilidade polínica em maracujazeiro-amarelo (Passiflora edulis sims f. flavicarpa degener). Lavras. Ciência e agrotecnologia. 26: 1209-1217. 2002. Disponível em: $<$ http://www.scielo.br/scielo.php?script=sci_nlinks\&ref=000152\&pid=S1807$8621201400020001200023 \&$ Ing=en>.

VARA PRASAD, P. V.; CRAUFURD, P. Q.; SUMMERFIELD, R. J.Fruit number in relation to pollen production and viability in Groundnut exposed to short episodes of heat stress. Annals of Botany, 84: 381-386.1999. Disponível em: <https://academic.oup.com/aob/article/84/3/381/2588072>. doi.org/10.1006/anbo.1999.0926 